\title{
Testing of a Microanalysis System
}

\author{
Hans G. Kerkhoff, Member, IEEE
}

\begin{abstract}
During the testing of microsystems, one has to cope with many problems resulting from inaccessibility, different technologies, and nonelectrical failure modes. A number of mixed-signal test techniques have been applied to test a new advanced microsystem. The choices on testing are directly dependent on implementation form and application area of the microsystem. Mixed-signal testing approaches are a key factor in this environment.
\end{abstract}

Index Terms-Fluidics system testing, microsystem testing, mixed-signal testing.

\section{INTRODUCTION}

$\mathbf{T}$ HE miniaturization of complete microsystems is developing at a very rapid pace. This not only results from new manufacturing techniques for sensors and actuators in many areas, such as fluidics (e.g., using MEMS), but also achievements in computer-aided design and testing of microsystems [1]. This paper deals with a microsystem consisting of sensors, analog and digital signal processing, and actuators in the fluidic domain.

A brief introduction in the technology and applications of microsystems can be found in [2]. Microsystems usually involve many different implementation technologies. This explains why implementation forms, such as multichip modules (MCMs) are popular [3].

Microsystem testing incorporates more challenges than conventional electrical testing, as also embedded sensors, actuators, and special structures (e.g., pure mechanical) are involved. The idea behind this paper is based on the fact that, using indirect methods, mixed-signal tests can provide most of the required information to guarantee the quality of the microsystem. A direct result of the miniaturization of systems is a dramatically decreased accessibility and hence decreased testability of these systems. The testing in microsystems can include digital, analog/mixed-signal testing, sensor/actuator testing, and the testing of special structures.

With regard to testing the digital IC parts, a significant amount of work has already been carried out which can be directly used in microsystems. Digital multiplexing, digital busses, built-in self-testing (BIST) of regular structures, and a variety of (boundary)-scan techniques have been developed in the past [4]. In the digital signal-processing chips, boundary-scan (library) cells can be integrated. If not available within the dies, this hardware can be incorporated in the (active) silicon substrate of a MCM [5].

Manuscript received March 1, 2001; revised July 30, 2001.

The author is with MESA+ Research Institute, University of Twente, Testable Design and Test of Microsystems Group (TDT), 7500 AE Enschede, The Netherlands.

Publisher Item Identifier S 0018-9456(01)10924-1.
For analog and mixed-signal IC parts and SMDs, the situation is more complex and less matured. One approach makes use of existing data converters [analog-to-digital converters (ADCs)/digital-to-analog converters (DACs)] in the microsystem and the flexibility of the vast digital signal-processing capabilities. In addition, concepts such as (mixed-signal) boundary-scan [6] can be applied, while other approaches, such as analog busses or BIST techniques [7], are currently being investigated.

The front- and back-end of most microsystems are very difficult to test. This is because often, nonelectrical stimuli are required or nonelectrical responses have to be measured. Sometimes the required packaging for handling the physical quantities involved in the microsystem (e.g., a fluid) complicate the testing of the system even more. In several cases where these physical properties can be electrically generated/converted on chip or substrate, there are possibilities for self-testing. Examples of devices where electrical generation is potentially feasible include temperature, magnetic, and optical devices. In these cases, tests can be carried out in the electrical domain, which will usually involve analog/digital signals.

The most complex parts to test in a microsystem are usually the ones that do not involve any electrical signals at all. An example is a static fluid mixer, which will be discussed later in detail. This structure is purely mechanical, which enhances the diffusion process and which can, for example, be verified in indirect ways using additional sensors or actuators. Then again, this can be brought back to basically a mixed-signal testing problem. However, the costs in terms of overhead of these (sensor/actuator combinations) are high. We usually refer to additional structures to ease testing as Design-for-Test (DfT) structures. They are intended to be used for testing purposes only, and should not affect the functional behavior. More specifically, they should not decrease the performance in terms of speed.

As an example, the microanalysis system (MAS) [8]-[10] for evaluating reaction products resulting from mixed fluids will be investigated, and the requirements with regard to mixed-signal testing will be presented. The system can, in extended form, be used as a portable instant chemical analyzer of fluids (e.g., for measuring the quality of surface water).

\section{ARChitecture OF THE MicRoAnAlysis System}

In this section, the MAS is explained [8]. The system consists of three microfluid channels etched in a silicon substrate; two inlets and one outlet. After each of the inlets, a flow-sensor and a micropump are located. An optical absorption module is situated at the outlet. The overall scheme is shown in Fig. 1.

The system measures chemical reaction products after mixing of two fluids (sample and reagent) by optical detection of the 


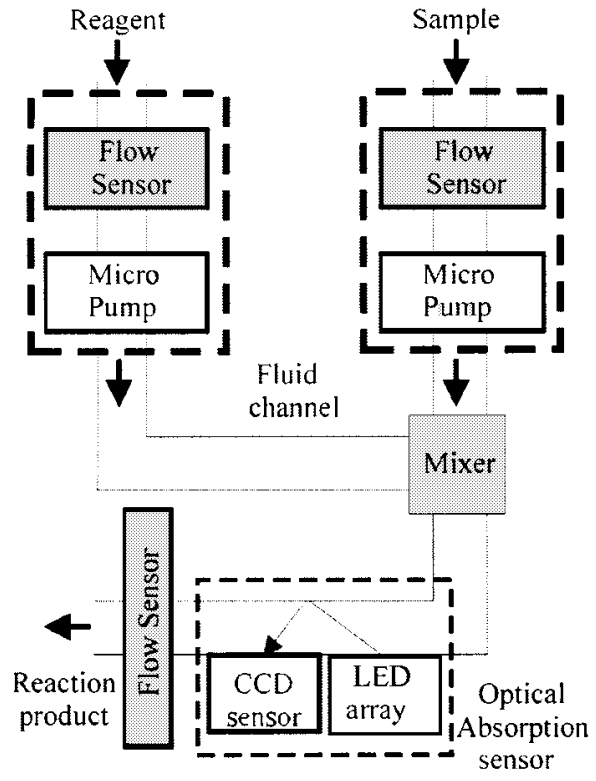

Fig. 1. Global setup of a part of the MAS system.

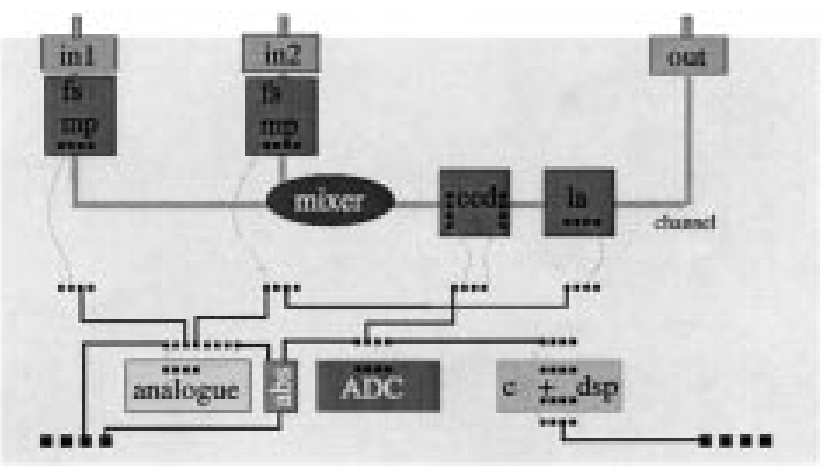

Fig. 2. MCM implementation of a single-reagent MAS.

spectral absorption intensity. As previously indicated, the physical implementation of a system has direct testing consequences. The implementation chosen in this paper is targeted to reduce the testing, diagnosis, and repair effort.

The digital, analog, and mixed-signal bare dies are glued on top of the silicon substrate in the region were no fluid channels are present (see Fig. 2). This is to circumvent electronic interconnection problems while crossing the fluid channels. Electrical crossing of channels required for the stand-alone flowsensor/micropump combinations is accomplished by using conventional wire-bonding techniques.

A microphotograph of an integrated flow sensor and a fluid channel is seen in Fig. 3(a) and a possible implementation of a micropump in Fig. 3(b). In the most advanced set-up, it is possible to merge the flow sensor and micropump as they require the same processing steps. This is very favorable from a testing and repairing point of view, as will be seen later.

The optical-absorption sensor consists of the combination of a small optical sensor [charge-coupled device (CCD)] and an optical source [a light-emitting device (LED) array], as shown in Fig. 1. In order to have both devices reside on top of the fluid channel, a reflection (mirror-layer) in the channel is required.

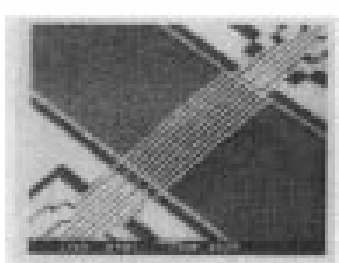

(a)

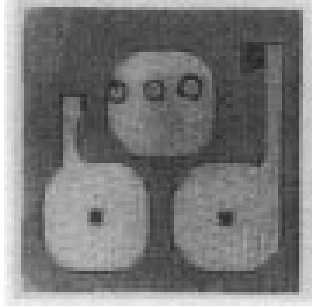

(b)
Fig. 3. (a) Resistor-based flow sensor with fluid channel in the silicon substrate. (b) The silicon, resistor-based micropump incorporating two mechanical valves.

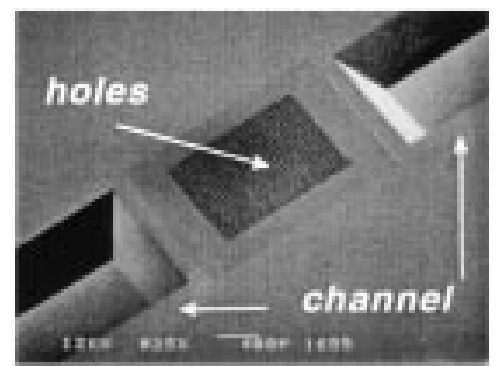

Fig. 4. Mechanical fluid mixer. Fluid 1 passes via the diagonal channel. Fluid 2 is injected via holes from the underside.

Otherwise, interconnection/packaging problems will arise. The (fluid) mixer module is a passive mechanical structure on top of the substrate of which a photomicrograph is shown in Fig. 4. The principle is based on enlarging the common diffusion area of both fluids by introducing one fluid via a number of microholes in the other fluid channel. The flow sensor at the reaction-product output (see Fig. 1) is not required functionally but is very useful to detect fluid leakage and channel/mixer jamming electronically.

In the MCM of Fig. 2, the fluid channels are etched on the top side of the silicon substrate topped with Pyrex with holes in it for the flow sensor, mixer, and flow-sensor/micropump combinations. On the bottom side of the figure, the metal-layer interconnections, bonding-paths for modular devices/bare dies are located. If required, (mixed-signal) DfT cells can be integrated (active MCM substrate) [5].

\section{Role OF MiXed-Signal Testing IN THE MICROANALYSIS SYSTEM}

The MAS test flow is based on the well-known good die (KGD) and known good substrate (KGS) test principle as common in MCM testing. However, depending on the MAS implementation, bare dies can also be micromechanical devices or the substrate can inherently house micromechanical devices.

With regard to the substrate, the fluid channels can be visually inspected for deformations or dust-particle jamming. If required, the holes can be temporarily closed by a thermal foil. This enables the use of fluids in the substrate for a functional test. By heating up the foil, it can be removed without causing any damage to the substrate. However, this procedure is usually not necessary.

In the case of a passive substrate (only interconnection wires and passive devices), one may require substrate interconnection 


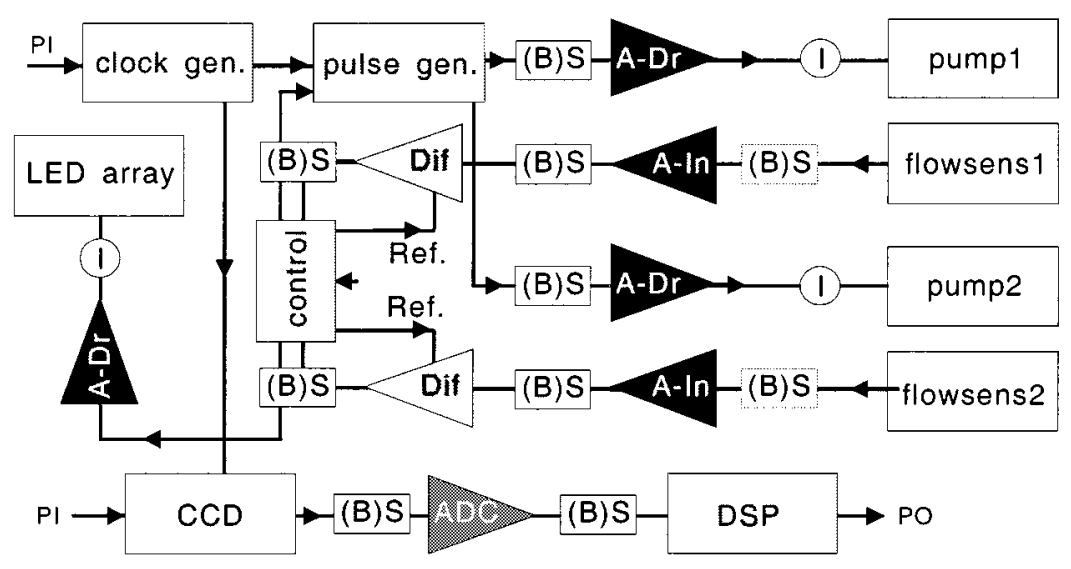

Fig. 5. Possible architecture of MAS and an extensive use of DfT structures.

tests, and resistance tests for the electrical integrity of the passive devices. Capacitive tests can verify the correct operation of integrated capacitors. In the case of integrated DfT structures (active substrate), wafer tests have to be carried out first, employing the substrate bonding pads. These include the MCM substrate pads in combination of the total package and the ones required for bonding the bare dies on the substrate.

The next step is the parametric or functional testing of the sensors, actuators, and special devices. As the flow-sensors and micropumps are both resistor-based, a simple parameter test can already reveal many problems. Opens, shorts, and incorrect resistance values indicate a failing device. However, they do not necessarily indicate a correct device, as the integrated fluid channels or mechanical valves can be jammed. For this, a complete functional test is required including correct electrical signals and fluids. An alternative is to use defect-oriented testing for detecting defects in the electronics as well as the fluidics part of the sensors and actuators. This involves the development of faults models which can be handled by conventional microelectronic CAD tools. As has been shown in previous works [11]-[14], this can lead to interesting "test-patterns" for fluidic components, not necessarily being functional tests.

Again using a thermal foil, which can be easily connected and removed by applying heat, together with a test fluid channel, can rule out these faults. It is even more preferable to connect the sensors/actuators immediately to a correct substrate, as they can be easily removed after heating up the foil and, in addition, verify the (channel) hole alignments.

A combined flow-sensor/micropump device is not only ideal because they use the same manufacturing process, but also for electrical testing purposes as the combination tests both devices at the same time in the same (electrical) domain. A mixed-signal verification tester has been used, which in fact now emulates part of the system in this approach.

A special device such as the mixer can only be visually inspected or functionally tested and replaced using the same previous (foil) procedure. This can only be carried out using an indirect test method using (optical-electrical) equipment to determine the degree of fluid mixing, using reference fluids.

The following step is the assembly of the optical absorption unit (see Fig. 1). Before assembly, these bare-die devices have been functionally verified separately. The LED/laser array and
CCD can be tested purely functional and are handled as bare dies, using, for example, conductive foils for interconnection. Light emission and associated currents are verified in the LED array. The CCD is tested as digital shift-register and as light/dark sensor. As repair of these devices after assembly is also rather difficult because of the transparent layer and the transparency deterioration after repair of this layer, a combined functional test is also carried out afterwards. The following sensor/actuator test involved is verifying the optical absorption module. The CCD shift operation is tested by pure electronic methods. The LED array is not powered during this operation. Next, the LED array is sequentially activated and the response of the CCD compared digitally. The LED array activation can be monitored separately by measuring the power current (see Fig. 5).

As all other dies are mounted on this combined substrate later, with the option of relative easy repair, the correct operation and testing of the sensors/actuators/substrate combination (including electrical and nonelectrical properties) is vital. The following step is the assembly of all digital and analog bare dies.

The digital chip (light-gray shading), consisting of the clock generator, pulse generator, control, and DSP block is tested using structural ATPG and (boundary) scan techniques [4] ((B)S) as well as functional tests (see Fig. 5). As this chip is locally designed, the boundary-scan cells are incorporated in the chip. Structural ATPG-based tests as well as (low-frequency) functional tests can be carried out at the bare die level.

There are two analog/mixed-signal chips (black and half-black boxes). One incorporates the amplifying drivers (A-Dr) and amplifying integrators (A-In) and differential amplifiers (Dif). If locally designed, this chip is equipped with mixed-signal boundary-scan cells [6] and current sensors [11] (seeFig. 5). If externally bought, active substrate DfT structures are recommended (Fig. 6). The ADC can be bought as bare die and is typically not equipped with any DfT. In addition, in this case it is possible is to incorporate mixed-signal boundary-scan cells in the active substrate [5], located around this bare die (Fig. 6). The bare dies can be tested with standard analog functional tests and standard, DSP-based ADC tests, again using conductive foils.

As Fig. 5 shows, all interconnections between the chips, including wire-bondings after assembly, can be tested using the boundary-scan cells. The same holds for the structural integrity 


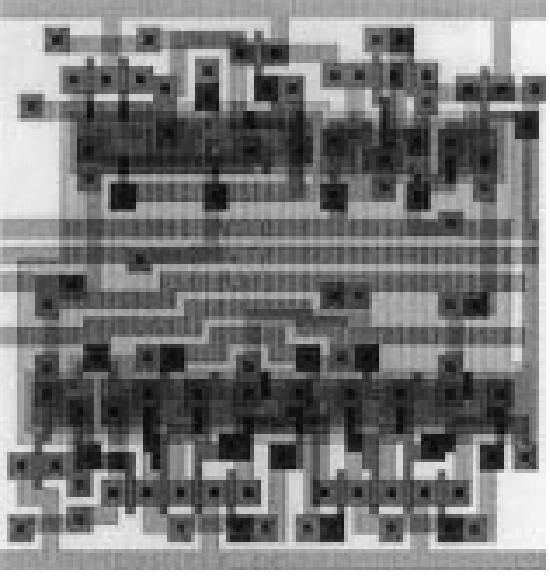

Fig. 6. Example of an MCM active substrate ABS cell.

of the bare dies on the substrate. A selection of previous bare-die tests can be chosen subsequently for structural or functional tests. Any repair on chips and/or wire-bondings should be completed.

Now, the functional testing of the total microsystem behavior remains. As this is often a standard customer requirement, it is hard to circumvent, even in the case of significant DfT support. Although the previous DfT will make detailed diagnostics at the module level easy, as an alternative, only overall functional tests can sometimes indicate problems. The parallel set-up of the inlets of this microsystem (see Fig. 1) is utilized for testing after complete assembly, using actual fluids. First, only the left branch is verified/activated, followed by the right branch. The combined flow-sensor and micropump, and the optical absorption module or outlet flow-sensor, can determine whether the absorption module, flow sensors, or micropump are malfunctioning. These tests also verify correct interconnections between the sensors/actuators and the electronics, and unobstructed passage in the fluid channels and mixer or leakage which are strictly mechanical processes. Jamming of the fluid mixer caused by dust particles, as well as leakage in the channels, can be indirectly tested with a flow sensor (see Fig. 1).

If the optical module response differs from the (standard) reagent fluid value, then the LED, CCD, or reflecting channel causes problems. If this is not the case, and the flow sensor does not provide any signal, then the inlet flow sensor is not correct. As actuators often consume power (such as a micropump or LED), an absence of increased current during operation can also be used for diagnostics. These tests are repeated for the other branch. It is assumed that the control electronics of the sensors and actuators has already been verified in an early stage.

Although being an example of a professional microsystem, only a fraction of the above tests will be carried out in the production. It is further noted that the functionality of some of the mechanical parts is not explicitly tested. Any jamming of the microfluid channels (e.g., by dust particles), or failing micropump valves will appear as failing flow sensor and pump operation.

In the case of separate substrate-integrated flow-sensors and micropumps, the testing, diagnostics, and repair approach would be completely different for this microsystem as previously discussed.

\section{CONCLUSIONS AND RECOMMENDATIONS}

Many digital, analog, and mixed-signal test methodologies and support hardware and software have been developed in the past for ICs and PCBs. A significant portion of these techniques can also be applied to microsystems. Although developments in digital parts are well matured, research efforts are still required with regard to analog and mixed-signal testing. This is especially important for microsystems. A large amount of work is still required with regard to testing the sensor, actuator, and special device parts of microsystems. In particular, the modeling and simulation of microfluidic components still requires a lot of research. Some devices lend themselves for BIST, enabling testing in the mixed-signal domain. Others require external stimuli and evaluation and even substrate assembly before testing. However, nonelectrical failures can often be indirectly measured in electrical (mixed-signal) quantities. The costs for transformation in the electrical domain for testing purposes can be high. For sensors and actuators, mixed-signal boundary-scan techniques in combination with powerful on-board DSP, enabling BIST, is expected to be the major microsystem test approach in the future. It turns out that the test approaches used are to a great extend dependent on the physical implementation and application area of microsystems.

\section{ACKNOWLEDGMENT}

The author would like to thank H. Hendriks, E. Oostenbroek, and A. van der Berg for contributing to this research in several ways.

\section{REFERENCES}

[1] B. Courtois and S. Mir et al., "CAD tools and foundries to boost microsystem development," Mat. Sci. Eng. B, vol. 51, pp. 242-253, 1998.

[2] J. Wilkinson, "Microsystems technology and applications," in Tutorial Booklet VLSI’97, Gramado, Brazil, 1997.

[3] H. G. Kerkhoff, "Testing of microsystems," in Proc. Microsystem Symp., Delft, The Netherlands, 1998, pp. 27-36.

[4] H. Bleeker et al., Boundary-Scan Testing: A Practical Approach. Norwell, MA: Kluwer, 1992.

[5] H. G. Kerkhoff et al., "MCM-D mixed-signal boundary-scan testing," in European Test Workshop, Cagliari, Italy, 1997, pp. 9.1-9.5.

[6] B. Wilkins, "1149.4 standard for a mixed-signal test bus," IEEE, Std., 1997.

[7] K. Arabi and B. Kaminska, "Testing analog and mixed-signal integrated circuits using oscillation test method," IEEE Trans. Computer-Aided Design, vol. 16, pp. 745-753, July 1997.

[8] A. van den Berg and P. Bergveld, "Development of MicroTAS concepts at the MESA Research Institute," in Proc. MicroTAS, 1996, pp. 9-15.

[9] A. C. Aarden et al., "Study of a testable microanalysis system," University of Twente, Enschede, The Netherlands, Rep. 060-2571, 1998.

[10] H. G. Kerkhoff, "Testing philosophy behind the microanalysis system," Proc. SPIE, vol. 3680, pp. 78-83, 1999.

[11] S. Bracho, "Dynamic current testing methods in mixed-signal integrated circuits," in Proc. ESPRIT “AMATIST" Open Workshop, Enschede, The Netherlands, 1997, pp. 6.1-6.9.

[12] H. G. Kerkhoff and H. P. A. Hendriks, "Fault modeling and fault simulation of mixed microfluidic microelectronic systems," JETTA, Fall 2001.

[13] H. G. Kerkhoff and E. Oosterbroek, "Defect-oriented testing of microelectronic/fluidic systems," in Proc. Int. Symp. Micro Total Analysis Systems, Enschede, The Netherlands, 2000, ISBN 0792363 876, pp. 315-319.

[14] H. G. Kerkhoff and H. P. A. Hendriks, "Analogue fault modeling and simulation techniques in electronic/fluidic microsystems," in Proc. 6th IEEE Int. Mixed-Signal Testing Workshop, Montpellier, France, June 2000, pp. 7-13. 


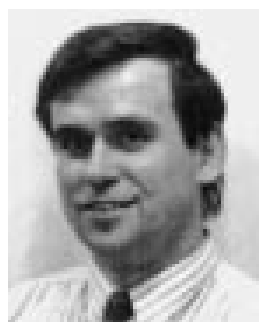

Hans G. Kerkhoff (M'99) received the M.Sc. degree in telecommunication (with honors) from the Technical University of Delft, Delft, The Netherlands, in 1977, and the Ph.D. degree in technical science (microelectronics) from the University of Twente, Enschede, The Netherlands, in 1984. While at the University of Delft, he received the Delft Hogeschool Award for his M.Sc. thesis.

In 1977, he became a Staff Member of the chair IC-Technology and Electronics at the Faculty of Electrical Engineering, University of Twente. In 1991, he became head of the group "Testable Design and Test of Microsystems" of the MESA Research Institute. In 1992, he spent his sabbatical year at the test company Advantest, San Jose, CA. From 1995 to 1999, he also worked part-time at the Philips Research Laboratories in the VLSI Design and Test Group, Eindhoven, The Netherlands. In 2000, he founded the company TwenTest, specializing in consultancy in the area of testable design and test of mixed-signal microsystems, and has advised eight Ph.D. students in this area. He has authored or coauthored over 100 publications. 